\title{
ON OPERATOR RANGES
}

\author{
BHUSHAN L. WADHWA
}

\begin{abstract}
If $f$ is any vector-valued bounded function defined on open set $D$ of the complex plane, and $T$ is any bounded linear operator on a Hilbert space such that $\sigma_{R}\left(T^{*}\right)$ is empty and if $(T-z I)^{2} f(z)=x$ for all $z$ in $D$ then $f$ is analytic.
\end{abstract}

Let $T$ be a bounded linear operator on a Hilbert space $H$. Let $f$ be an $H$-valued function defined on an open set $D$ of the complex plane. Suppose $(T-z I) f(z)=$ $x$ for all $z$ in $D$ and for a fixed $x$ in $H$. The question arises: What type of conditions on the operator $T$ and on the function $f$ will be sufficient to insure that $f$ is analytic on $D$ ? This question has been implicitly discussed in Clancey [1], Johnson [4], Putnam [5], [6], Radjabalipour [7], and Stampfli-Wadhwa [9], [10], under various conditions related to normality on $T$. One of the main results is: If $T$ is a hyponormal operator $\left(T T^{*}<T^{*} T\right)$ and $f$ is any function satisfying the above equation then $f$ is analytic in $D$. See [1]. On the other hand, there is a cohyponormal operator $T,\left(T^{*}\right.$ is hyponormal) and a bounded function $f$ satisfying the above equation which fails to be analytic on $D$. See [6].

In this note, by a simple argument, we shall show that if $(T-z I)^{2} f(z)=x$ for all $z$ in $D$ and if $f$ is bounded on $D$ and $\sigma_{R}\left(T^{*}\right)=\varnothing$ (range of $T^{*}-\bar{z} I$ is dense in $H$ for all $z$ ) then $f$ is analytic. We shall use this result to give an alternative proof of a classical result of Stampfli [8] about quadratically hyponormal operators.

The following lemma is implicitly contained in most of the references mentioned previously. We include it for the sake of completeness.

LEMMA. Let $(T-z I) f(z)=x$ for all $z$ in $D$ be such that $f$ is bounded and $\sigma_{R}\left(T^{*}\right)=\varnothing$ then $f$ is weakly continuous.

Proof. For any $z$ and $z_{0}$ in $D$,

$$
\begin{aligned}
\left(f(z)-f\left(z_{0}\right),\left(T^{*}-\bar{z}_{0} I\right) y\right) & =\left(\left(T-z_{0} I\right)\left(f(z)-f\left(z_{0}\right)\right), y\right) \\
& =\left(z-z_{0}\right)(f(z), y) \text { for all } y \text { in } H .
\end{aligned}
$$

Since $f$ is bounded and range of $\left(T^{*}-\bar{z}_{0} I\right)$ is dense in $H$, it follows that $f$ is weakly continuous.

THEOREM. Let $g$ be a bounded vector-valued function such that $(T-z I)^{2} g(z)=x$ for all $z$ in $D$ and let $\sigma_{R}\left(T^{*}\right)=\varnothing$. Then $g$ is analytic on $D$.

Proof. Let $f(z)=(T-z I) g(z)$. Then $(T-z I) f(x)=x$ for all $z$ in $D, f$ is bounded and hence weakly continuous on each bounded subset $D_{0}$ of $D$. Now for

Received by the editors October 30, 1979 and, in revised form, January 29, 1980.

AMS (MOS) subject classifications (1970). Primary 47A05, 47B20. 
any $z$ and $z_{0}$ in $D_{0}$,

$$
\left(\left(f(z)-f\left(z_{0}\right)\right) /\left(z-z_{0}\right),\left(T^{*}-\bar{z}_{0} I\right) y\right)=(f(z), y),
$$

and

$$
\begin{aligned}
\lim _{z \rightarrow z_{0}}\left(\left(f(z)-f\left(z_{0}\right)\right)\right. & \left./\left(z-z_{0}\right),\left(T^{*}-\bar{z}_{0} I\right) y\right)=\left(f\left(z_{0}\right), y\right) \\
= & \left(\left(T-z_{0} I\right) g\left(z_{0}\right), y\right)=\left(g\left(z_{0}\right),\left(T^{*}-\bar{z}_{0} I\right) y\right)
\end{aligned}
$$

for all $y$ in $H$.

Since range of $\left(T^{*}-\bar{z}_{0} I\right)$ is dense in $H, f$ is analytic and $f^{\prime}(z)=g(z)$ for all $z$ in D.

Let $T$ be a quadratically hyponormal operator $\left(a T^{2}+b T+c I\right.$ is hyponormal for all complex numbers $a, b$ and $c)$. Let $\rho(T, x)$ be the local resolvent of the vector $x$ with respect to the operator $T$ (see Dunford and Schwartz [3, p. 1935]).

CoROllaRY (STAMPfli [8]). If $T$ is a quadratically hyponormal operator with $\sigma_{R}(T)=\varnothing$ then $\rho(T ; x) \subset \overline{\rho\left(T^{*}, x\right)}$. (The bar denotes the complex conjugate of the set.)

Proof. Let $z_{0} \in \rho(T, x)$; thus there exists an analytic function defined on a bounded set $D$ containing $z_{0}$ such that $(T-z I) f(z)=x$. Since $f$ is analytic, a simple computation shows that $(T-z I)^{2} f^{\prime}(z)=x$ for all $z$ in $D$. Since $T$ is quadratically hyponormal, $(T-z I)^{2}\left(T^{*}-\bar{z} I\right)^{2}<\left(T^{*}-\bar{z} I\right)^{2}(T-z I)^{2}$. By Douglas [2], there is a contraction $K(z)$ such that $(T-z I)^{2}=\left(T^{*}-\bar{z} I\right)^{2} K(z)$. Consequently, $\left(T^{*}-\bar{z} I\right)^{2} g(\bar{z})=x$ where $g(\bar{z})=K(z) f^{\prime}(z)$. Thus $g(\bar{z})$ is bounded for $z \in D$. Using the Theorem we conclude that $g(z)$ is analytic for all $z$ in $\bar{D}$ and $\left(T^{*}-z I\right)\left(T^{*}-z I\right) g(z)=x$ for all $z$ in $\bar{D} ;$ thus $z_{0} \in \overline{\rho\left(T^{*}, x\right)}$.

\section{REFERENCES}

1. K. Clancey, On local spectra of seminormal operators, Proc. Amer. Math. Soc. 72 (1978), 473-479.

2. R. G. Douglas, On majorization, factorization and range inclusion of operators on Hilbert space, Proc. Amer. Math. Soc. 17 (1966), 413-415.

3. N. Dunford and J. Schwartz, Linear operators. III: Spectral operators, Pure and Appl. Math., Vol. VII, Interscience, New York-London-Sydney, 1971.

4. B. E. Johnson, Continuity of linear operators commuting with continuous linear operators, Trans. Amer. Math. Soc. 128 (1967), 88-102.

5. C. R. Putnam, Ranges of normal and subnormal operators, Michigan Math. J. 18 (1971), 33-36.

6. , Hyponormal contractions and strong power convergence, Pacific J. Math. 57 (1975), $531-538$.

7. M. Radjabalipour, Ranges of hyponormal operators, Illinois J. Math. 21 (1977), 70-75.

8. J. G. Stampfli, Analytic extensions and spectral localization, J. Math. Mech. 19 (1966), 287-296.

9. J. G. Stampfli and B. L. Wadhwa, An asymmetric Putnam-Fuglede theorem for dominant operators, Indiana Univ. Math. J. 25 (1976), 359-365.

10. __ On dominant operators, Monatsh. Math. 84 (1977), 143-153.

Department of Mathematics, Cleveland State University, Cleveland, Oho 44115 